\title{
A New Discrete Family of Reduced Modified Weibull Distribution
}

\section{Ademola Lateef Oloko, Osebekwin Ebenezer Asiribo, Ganiyu Abayomi Dawodu, Mathew Omonigho Omeike, Nurudeen Ayobami Ajadi", Abayomi Olumuyiwa Ajayi}

Department of Statistics, College of Physical Sciences, Federal University of Agriculture, Abeokuta, Ogun, Nigeria

Email address:

ajadinurudeen2014@yahoo.com (N. A. Ajadi)

${ }^{*}$ Corresponding author

\section{To cite this article:}

Ademola Lateef Oloko, Osebekwin Ebenezer Asiribo, Ganiyu Abayomi Dawodu, Mathew Omonigho Omeike, Nurudeen Ayobami Ajadi, Abayomi Olumuyiwa Ajayi. A New Discrete Family of Reduced Modified Weibull Distribution. International Journal of Statistical Distributions and Applications. Vol. 3, No. 3, 2017, pp. 25-31. doi: 10.11648/j.ijsd.20170303.11

Received: August 16, 2017; Accepted: August 31, 2017; Published: October 26, 2017

\begin{abstract}
Discretization of continuous lifetime distribution is an interesting and intuitively appealing approach to derive a discrete lifetime model. This study derived a discretized form of Reduced Modified Weibull distribution known as the Marshall-Olkin Discrete Reduced Modified Weibull (MDRMW) distribution. The mathematical and statistical properties of MDRMW distribution were derived and compared with existing distributions of Discrete Reduced Modified Weibull distribution (DRMW), Exponentiated Discrete Weibull distribution (EDW) and Two Parameters Discrete Lindley distribution (TDL). Maximum likelihood method was used to derive the statistics of MDRMW parameters. The Aarset Reliability dataset was fitted for the existing and derived distribution and AIC and Kolmogorov Smirrnoff (KS) were compared. The shape of MDRMW distribution was unimodal and monotonic decreasing. The plot of hazard rate function could be decreasing or bathtub. The AIC and KS values of Aarset reliability data analysis were 483.9 and $0.17579 ; 507.8$ and $0.24435 ; 485.2$ and 0.17897 for MDRMW, DRMW and TDL respectively. The AIC and KS values of Leukemia survival data analysis were 668.2 and $0.11053 ; 751.9$ and 0.39285 respectively. The Aarset reliability data analysis showed that MDRMW compared favorably with existing distributions. The MDRMW and DRMW handled Leukemia survival data set as against EDW and TDL. The values of AIC and KS for MDRMW were lower than DRMW, EDW and TDL. This showed that MDRMW was better than the existing distributions.
\end{abstract}

Keywords: Weibull, TDL, DRMW, EDW, MDRMW

\section{Introduction}

The Weibull distribution is a lifetime distribution, this make it to be important and desirable. Weibull distribution can be used in different fields with many applications. Its survival and hazard rate functions have simple expression and its flexibility make it useful to fit different lifetime data sets in different fields.

The cumulative distribution function (CDF) of the twoparameter Weibull distribution is given by the

$$
F(x)=1-\exp \left(-\alpha_{x} \theta\right), \quad x>0
$$

Where $\alpha>0$, and $\theta>0$ are the scale and shape parameters respectively.

The probability density function (PDF) is given by

$$
f(x)=\alpha \theta x^{\theta-1} \exp (-\alpha x \theta), \quad x>0 .
$$

And the hazard rate function is

$$
h(x)=\alpha \theta x^{\theta-1}, \quad x>0,
$$

This can either increase; decrease or constant depending on $\theta>1, \theta<1$ or $\theta=1$. For many years, using different techniques, many researchers have developed various modified forms of the Weibull distribution to achieve nonmonotonic shapes. Bebbington et al (2007) proposed that the hazard rate function of the two-parameter flexible Weibull 
extension can be increasing, decreasing or bathtub shaped. Also Mudholkar and Srivastave (1993) proposed a twoparameter model, called the exponentiated Weibull distribution.

The authors are interested in using the Marshal-Olkin discretized family to discretize the modified Weibull distribution (MW) due to the wide ability to discretize any continuous distribution unlike the general method being used. There is no limit at which the method can discretize continuous distributions in as much as the continuous distribution has a survival function. Also, a shape parameter from the Marshal-Olkin family is an added advantage in the analysis of data.

The proposed distribution is referred to as Marshall-Olkin Discrete Reduced Modified Weibull distribution (MDRMW). The statistical properties of the proposed distribution were derived. The proposed distribution is compared with existing competing distributions and applied to medical and reliability The new distribution has a desired characteristic, it has a closed form and it can be highly monotonically decreasing in shape, its hazard function can be bathtub and it is useful in modeling medical and reliability data.

Marshall-Olkin Discrete Family

A methodology to add a parameter to obtain a new family of distributions was introduced by Marshall and Olkin (1997) The family of Marshall - Olkin distributions with survival function given as

$$
S(x ; \beta)=\frac{\beta \bar{F}(x)}{1-\bar{\beta} \bar{F}(x)^{\prime}}
$$

where $\beta>0, \bar{\beta}=1-\beta$ and $\bar{F}(x)$ is a survival function of the continuous distribution.

Here, the authors consider the class of continuous scale family of distributions with scale parameter $\sigma>0$ and denote probability density function and the cumulative distribution function of the same by $f(. \mid \sigma)$ and $F(. \mid \sigma)$ respectively. The survival function of the new scale family of continuous distributions on the positive real line using equation (4) is given by

$$
S(x ; \beta, \sigma)=\frac{\beta \bar{F}(x \mid \sigma)}{1-\bar{\beta} \bar{F}(x \mid \sigma)}
$$

where $\bar{F}(x \mid \sigma)=1-F(x \mid \sigma)$.

The survival function (5) can be considered as a generalization of the scale family of distributions and the corresponding family of distributions.

Let $X$ be a discrete random variable associated to a continuous random variable belonging to RMW.

The probability mass function is given by

$$
\begin{aligned}
& P(X=x ; \beta)=p_{x} \\
& p_{x}=S(x ; \beta)-S(x+1 ; \beta) \\
& p_{x}=\frac{\beta[\bar{F}(x)-\bar{F}(x+1)]}{(1-\bar{\beta} \bar{F}(x))(1-\bar{\beta} \bar{F}(x+1))}, x=0,1,2, \ldots
\end{aligned}
$$

where $\beta>0, \bar{\beta}=1-\beta$

\section{Methodology}

A two-parameter discrete distribution will be introduced. It will be a new discrete distribution allowing for bathtub shaped hazard rate functions. Its mathematical properties will be discussed, and its applications to real data sets will be studied. The proposed distribution will be shown to outperform the existing models including the ones allowing for bathtub shaped hazard rate functions.

The proposed discrete distribution is based on a twoparameter modification of the Reduced Modified Weibull (RMW) distribution proposed by Almaki, (2014). The twoparameter distribution would be shown to be flexible, have a bathtub shaped hazard rate function. The authors refer to it as the Marshall-Olkin Discrete Reduced Modified Weibull distribution and denoted by MDRMW.

\section{Proposed Distribution: Marshall-Olkin Discrete Reduced Modified Weibull Distribution (MDRMW)}

The cumulative distribution function and survival function of RMW distribution are respectively as follows

$$
F(x)=1-e^{-\alpha x^{1 / 2}-\delta x^{1 / 2} e^{\lambda x},}
$$

and

$$
\bar{F}(x)=e^{-\alpha x^{1 / 2}-\delta x^{1 / 2} e^{\lambda x}},
$$

respectively.

$$
p_{x}=\frac{\beta\left(q^{x^{1 / 2}\left(1+b c^{x}\right)}-q^{(x+1)^{1 / 2}\left(1+b c^{x+1}\right)}\right)}{\left(1-\bar{\beta} q^{x^{1 / 2}\left(1+b c^{x}\right)}\right)\left(1-\bar{\beta} q^{(x+1)^{1 / 2}\left(1+b c^{x+1}\right)}\right)}
$$

Equation (9) is referred to as Marshall-Olkin Discrete Reduced Modified Weibull distribution (MDRMW) which is the proposed model.

\subsection{Properties of Proposed Distribution (MDRMW)}

The cumulative distribution function and survival function of the discrete random variable having the probability mass function (9) is given by

$$
F(x ; \beta, q, b, c)=\frac{1-q^{(x+1)^{1 / 2}\left(1+b c^{x+1}\right)}}{1-\bar{\beta} q^{(x+1)^{1 / 2}\left(1+b c^{x+1}\right)}}, x=0,1,2, \ldots
$$

and

$$
S(x ; \beta, q, b, c)=\frac{\beta q^{x^{1 / 2}\left(1+b c^{x}\right)}}{1-\bar{\beta} q^{x^{1 / 2}\left(1+b c^{x}\right)}}, x=0,1,2, \ldots
$$

\subsection{Shape of the Proposed Distribution (MDRMW)}

In Figure 1, it can be seen that the MDRMW distribution is flexible. Its PMF can take one of the following shapes: i) a unimodal shape; ii) a monotonic decreasing shape; iii) a 
decreasing shape followed by an increasing shape followed by a decreasing.

\section{Shape of MDRMW}

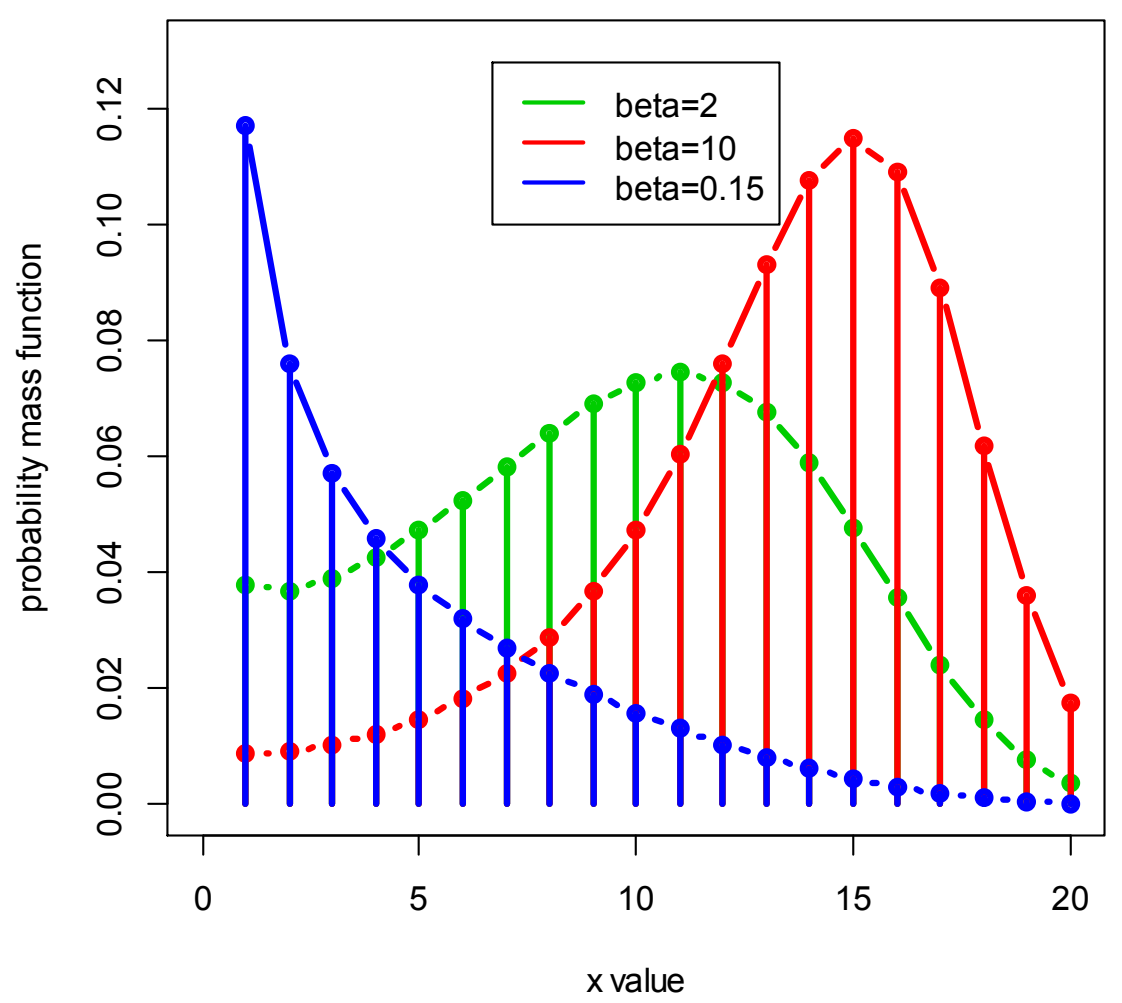

Figure 1. The shape of pmf of the proposed distribution.

\subsection{Series Expansion of the Proposed Distribution}

Here, a series expansion for the survival function is derived, and hence for the probability mass function. Using the series expansion for the exponential, it can be written as

$$
\begin{gathered}
q^{x^{1 / 2}\left(1+b c^{x}\right)} \\
\sum_{j=0}^{\infty} \frac{\ln ^{j}(q) x^{j / 2}}{j !} \sum_{k=0}^{j}\left(\begin{array}{l}
j \\
k
\end{array}\right) b^{k}\left(\sum_{i=0}^{\infty} \frac{x^{i} l n^{i} c}{i !}\right)^{k}
\end{gathered}
$$

Now using the partial exponential Bell polynomial, $B_{r k}(x)$ (Comtet, 1974), defined by

$$
\begin{aligned}
& \left(\sum_{r=1}^{\infty} x_{r} t^{r} / r !\right)^{k} / k !=\sum_{r=k}^{\infty} B_{r k}(x) t^{r} / r ! \\
S(x)= & \sum_{j=0}^{\infty} \frac{\ln ^{j}(q) x^{j / 2}}{j !} \sum_{k=0}^{j}\left(\begin{array}{l}
j \\
k
\end{array}\right) b^{k} k ! \sum_{l=k}^{\infty} B_{r k}(\ln c, \ln c, \ldots) \frac{x^{l}}{l !} \\
= & \sum_{j}^{\infty} \sum_{k}^{j} \sum_{l=k}^{\infty} \frac{\ln ^{j}(q) x^{j / 2} B_{r k}(\ln c, \ln c, \ldots)}{(j-k) l !} x^{l+\frac{j}{2}} .
\end{aligned}
$$

The representation in (13) can be used to derive similar expansions for the probability mass function as the following

$$
\beta\left(\sum_{j}^{\infty} \sum_{k}^{j} \sum_{l=k}^{\infty} \frac{\ln ^{j}(q) x^{j / 2} B_{r k}(\ln c, \ln c, \ldots)}{(j-k) l !} x^{l+\frac{j}{2}}-\sum_{j}^{\infty} \sum_{k} \sum_{l=k}^{\infty} \frac{\ln ^{j}(q) x^{j / 2} B_{r k}(\ln c, \ln c, \ldots)}{(j-k) l !}(x+1)^{l+\frac{j}{2}}\right)
$$




$$
\left(1-\bar{\beta} \sum_{j=0}^{\infty} \sum_{k=0}^{j} \sum_{l=k}^{\infty} \frac{\ln ^{j}(q) x^{j / 2} B_{r k}(\ln c, \ln c, \ldots)}{(j-k) l !} x^{l+\frac{j}{2}}\right)\left(1-\bar{\beta} \sum_{j=0}^{\infty} \sum_{k=0}^{j} \sum_{l=k}^{\infty} \frac{\ln ^{j}(q) x^{j / 2} B_{r k}(\ln c, \ln c, \ldots)}{(j-k) l !}(x+1)^{l+\frac{j}{2}}\right)
$$

using the binomial expansion for non-integer powers:

$$
(A+B)^{P}=\sum_{d=0}^{\infty} c(p, d) A^{p-d} B^{p},
$$

where $c(p, d)=\frac{p(p-1)(p-2) \ldots(p-d+1)}{d ! !}$,

$$
\begin{aligned}
\varphi & =\beta\left(\sum_{j=0}^{\infty} \sum_{k=0}^{j} \sum_{l=k}^{\infty} \frac{\ln ^{j}(q) x^{j / 2} B_{r k}(\ln c, \ln c, \ldots)}{(j-k) l !} x^{l+\frac{j}{2}}-\sum_{j=0}^{\infty} \sum_{k=0}^{j} \sum_{l=k}^{\infty} \sum_{p=0}^{\infty} \frac{c\left(\frac{j}{2}+l, p\right) \ln ^{j}(q) x^{j / 2} B_{r k}(\ln c, \ln c, \ldots)}{(j-k) l !} x^{\frac{j}{2}+l-p}\right) \\
\omega & =\left(1-\bar{\beta} \sum_{j}^{\infty} \sum_{k}^{j} \sum_{l=k}^{\infty} \frac{\ln ^{j}(q) x^{j / 2} B_{r k}(\ln c, \ln c, \ldots)}{(j-k) l !} x^{l+\frac{j}{2}}\right)\left(1-\bar{\beta} \sum_{j}^{\infty} \sum_{k}^{j} \sum_{l=k}^{\infty} \sum_{p=0}^{\infty} \frac{c\left(\frac{j}{2}+l, p\right) \ln (q) x^{j / 2} B_{r k}(\ln c, \ln c, \ldots)}{(j-k) l !} x^{\frac{j}{2}+l-p}\right)
\end{aligned}
$$

Then

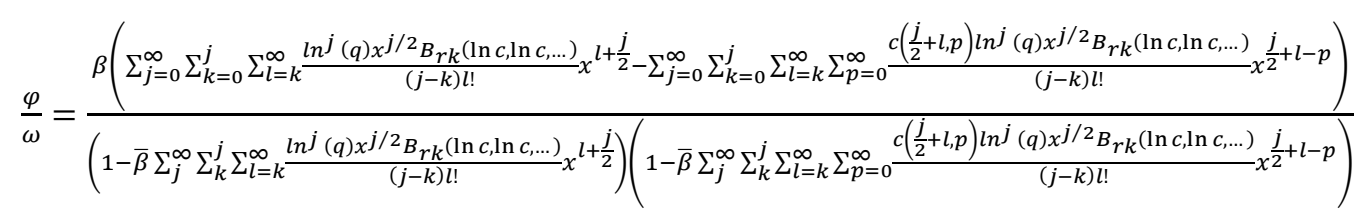

\subsection{Hazard Rate Function of the Proposed Distribution}

The Hazard rate function is

$$
\begin{gathered}
\frac{p(x)}{S(x)^{\prime}} \\
h(x)=\frac{q^{x^{1 / 2}\left(1+b c^{x}\right)}-q^{(x+1)^{1 / 2}\left(1+b c^{x+1}\right)}}{q^{x^{1 / 2}\left(1+b c^{x}\right)}\left(1-\bar{\beta} q^{(x+1)^{1 / 2}\left(1+b c^{x+1}\right)}\right)} \\
\text { Shape of hazard function of MDRMw }
\end{gathered}
$$

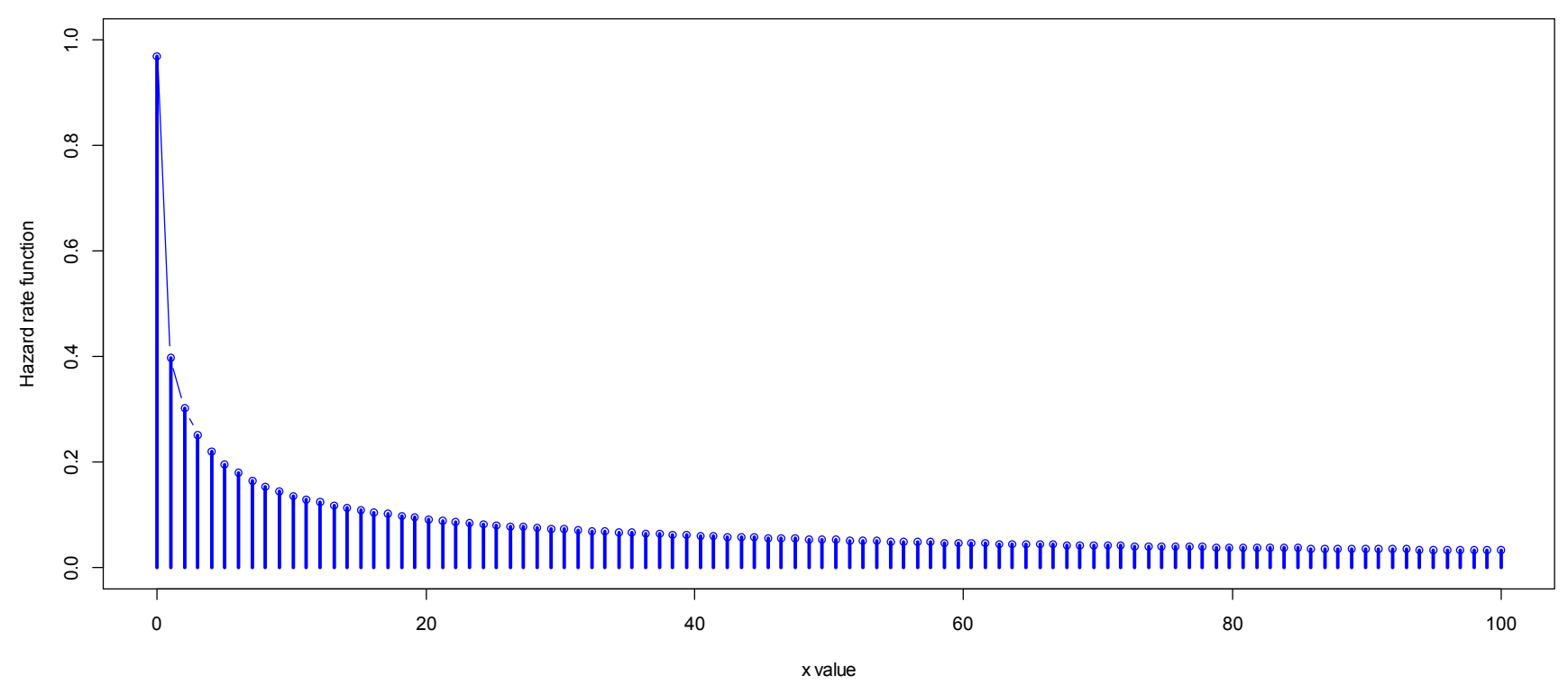

Figure 2. The decreasing hazard shape of the proposed distribution. 
Shape of hazard function of MDRMW

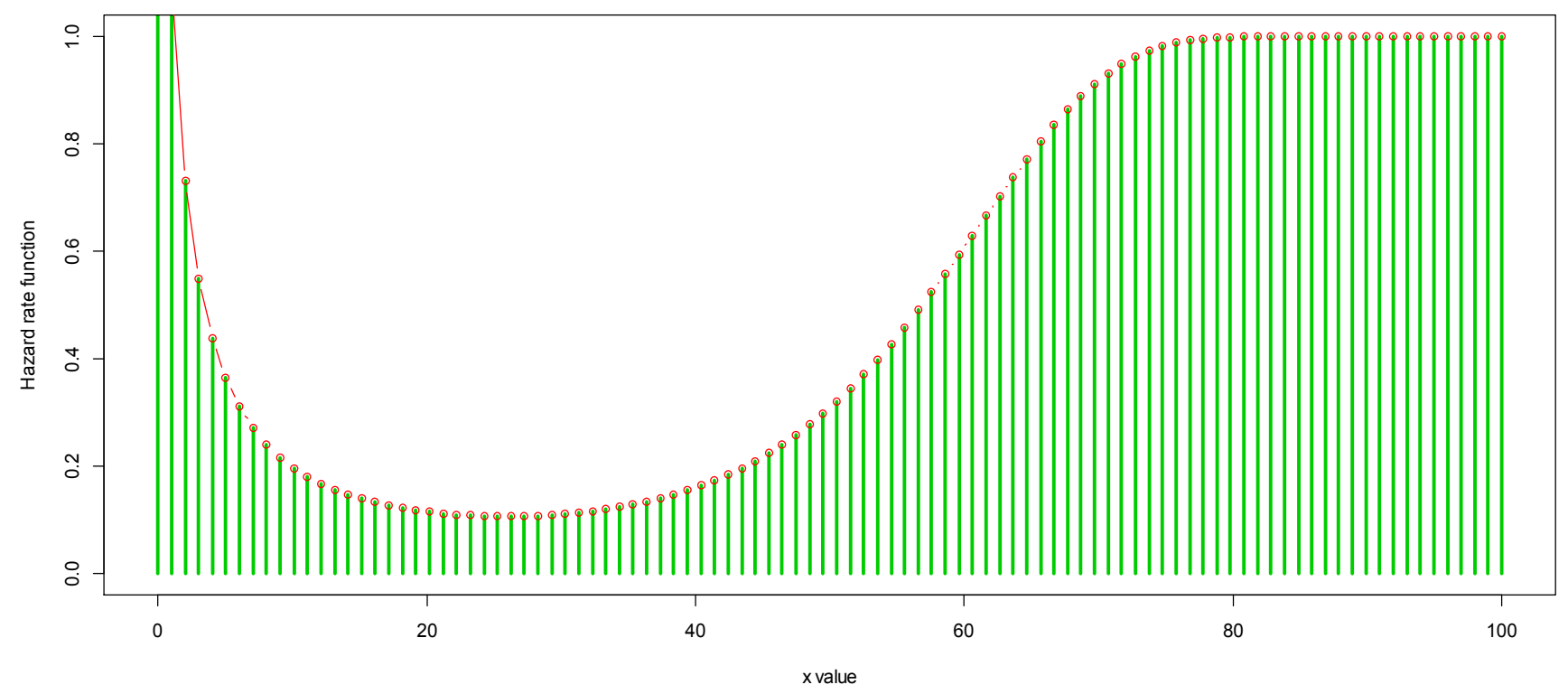

Figure 3. The decreasing then increasing hazard shape.

\subsection{The Probability Generating Function of the Proposed Distribution}

The probability generating function (pgf) of discrete random variable having the $\operatorname{MDRMW}(q, b, c, \beta)$ is given by

$$
\begin{aligned}
& p_{x}(t)=1+\beta(t-1) \sum_{x=1}^{\infty} t^{x-1} \frac{\bar{G}(x)}{1-\bar{\beta} \bar{G}(x)} \\
& =1+\beta(t-1) \sum_{x=1}^{\infty} t^{x-1} \frac{q^{x^{1 / 2}\left(1+b c^{x}\right)}}{1-\bar{\beta} q^{x^{1 / 2}\left(1+b c^{x}\right)}}
\end{aligned}
$$

mean and variance of the random variable having MDRMW $(q, b, c, \beta)$

$$
\begin{gathered}
\mu(q, b, c, \beta)=E(X)=\beta \sum_{x=1}^{\infty} \frac{q^{x^{1 / 2}\left(1+b c^{x}\right)}}{1-\bar{\beta} q^{x^{1 / 2}\left(1+b c^{x}\right)}} \\
V(X)=\beta \sum_{x=1}^{\infty}(2 x-1) \frac{q^{x^{1 / 2}\left(1+b c^{x}\right)}}{1-\bar{\beta} q^{x^{1 / 2}\left(1+b c^{x}\right)}}-\left(\beta \sum_{x=1}^{\infty} \frac{q^{x^{1 / 2}\left(1+b c^{x}\right)}}{1-\bar{\beta} q^{x^{1 / 2}\left(1+b c^{x}\right)}}\right)^{2}
\end{gathered}
$$

\subsection{Recurrence Relation of Probability Generating Function of the Proposed Distribution}

The recurrence relation for generating probabilities given by

$p_{x+1}=\frac{\bar{G}(x+1)-\bar{G}(x+2)}{\bar{G}(x)-\bar{G}(x+1)} \cdot \frac{1-\bar{G}(x)}{1-\bar{G}(x+2)} p_{x}, x=0,1,2 \ldots$

where $p_{0}=\frac{1-\bar{G}(1)}{1-\bar{\beta} \bar{G}(1)}$.

then,

$$
p_{x}=\frac{q^{(x+1)^{1 / 2}\left(1+b c^{x+1}\right)}-q^{(x+2)^{1 / 2}\left(1+b c^{x+2}\right)}}{q^{x^{1 / 2}\left(1+b c^{x}\right)}-q^{(x+1)^{1 / 2}\left(1+b c^{x+1}\right)}} \cdot \frac{1-\bar{\beta} q^{x^{1 / 2}\left(1+b c^{x}\right)}}{1-\bar{\beta} q^{(x+2)^{1 / 2}\left(1+b c^{x+2}\right)}} p_{x}, x=
$$

$0,1,2 \ldots$ where $p_{0}=\frac{1-q^{(1+b c)}}{1-\bar{\beta} q^{(1+b c)}}$

\subsection{Maximum Likelihood Estimation of the Proposed Distribution (MDRMW)}

The maximum likelihood estimation (MLE) is used to estimate unknown parameters. Consider a random sample $x_{1}, x_{2}, \ldots, x_{n}$ from the Marshall-Olkin Discrete Reduced Modified Weibull (MDRMW) distribution.

The likelihood function is given by

$$
\begin{gathered}
\log L=n \log \beta+\sum_{i=1}^{n} \log \left(q^{x_{i}{ }^{1 / 2}\left(1+b c^{x_{i}}\right)}-q^{\left(x_{i}+1\right)^{1 / 2}\left(1+b c^{x_{i}+1}\right)}\right)- \\
\sum_{i=1}^{n} \log \left(1-\bar{\beta} q^{x_{i}{ }^{1 / 2}\left(1+b c^{x_{i}}\right)}\right)- \\
\sum_{i=1}^{n} \log \left(1-\bar{\beta} q^{\left(x_{i}+1\right)^{1 / 2}\left(1+b c^{x_{i}+1}\right)}\right)
\end{gathered}
$$

Two applications using well-known data sets were used to demonstrate the robustness and applicability of the proposed model. These data present different degrees of skewness and kurtosis. The new distribution was compared with the existing distributions which are Discrete Reduced Modified Weibull distribution (DRMW) (Amalki, 2014), Two parameter Discrete Lindley distribution (TDL) (Tassaddaq et al., 2016).

\section{Numerical Illustration}

The goodness of fit statistic and the goodness of fit plot were provided in order to check the model that best fit the data among the models for each dataset used for this research.

Two real data sets were considered in this section. One of them has bathtub shaped hazard rate functions while the second one has an increasing hazard rate function. 


\subsection{Discrete Aarset Data}

The data are integer parts of the lifetimes of fifty devices. The data are listed in Table 1.
Nooghabi et al. (2011) had shown that the DMW distribution provides a good fit for this data.

Table 1. Aarset data (in weeks).

\begin{tabular}{|c|c|c|c|c|c|c|c|c|c|c|}
\hline Time of failure & 0 & 1 & 2 & 3 & 6 & 7 & 11 & 12 & 18 & 21 \\
\hline No of failure & 2 & 5 & 1 & 1 & 1 & 1 & 1 & 1 & 5 & 1 \\
\hline Time of failure & 32 & 36 & 40 & 45 & 46 & 47 & 50 & 55 & 60 & 63 \\
\hline No of failure & 1 & 1 & 1 & 1 & 1 & 1 & 1 & 1 & 1 & 2 \\
\hline Time of failure & 67 & 72 & 75 & 79 & 82 & 83 & 84 & 85 & 86 & \\
\hline No of failure & 4 & 1 & 1 & 1 & 2 & 1 & 3 & 5 & 2 & \\
\hline
\end{tabular}

Table 2 shows the Maximum Likelihood Estimation (MLEs) of the parameters and their standard errors. Table 3 shows the AIC and BIC values for the fitted MDRMW, DRMW, TDL and EDW distributions.

Table 2. The Maximum Likelihood Estimation of the MDRMW distribution for the Aarset Data and Standard Error.

\begin{tabular}{lcccl}
\hline MODEL & $\boldsymbol{q}$ & $\boldsymbol{\beta}$ & $\boldsymbol{b}$ & $\boldsymbol{c}$ \\
\hline \multirow{2}{*}{ MDRMW } & 0.6183 & 18.682 & 2.5905 & -0.1776 \\
& $(0.0443)$ & $(10.7933)$ & $(4.1552)$ & $(0.2084)$ \\
DRMW & 0.8475 & - & 7.4787 & -0.0817 \\
& $(0.0198)$ & - & $(22.5427)$ & $(0.2335)$ \\
TDL & 0.9687 & 0.0275 & - & - \\
\hline
\end{tabular}

Table 3. The AIC and BIC for the fitted distributions.

\begin{tabular}{llll}
\hline MODEL & -2Log-Likelihood & AIC & BIC \\
\hline MDRMW & 475.9 & 483.9 & 491.5 \\
DRMW & 501.8 & 507.8 & 513.5 \\
TDL & 481.2 & 485.2 & 489.0 \\
\hline
\end{tabular}

\subsection{Leukemia Data}

The data set for this example was collected from the Ministry of Health Hospital in Saudi Arabia. The data are lifetimes in days of forty three blood patients who had leukemia. The data set exhibits an increasing hazard rate.

Table 4. The Leukemia data.

\begin{tabular}{|c|c|c|c|c|c|c|c|c|c|c|c|}
\hline 115 & 181 & 255 & 418 & 441 & 461 & 516 & 739 & 743 & 789 & 807 & 865 \\
\hline 1062 & 924 & 983 & 1025 & 1063 & 1165 & 1191 & 1222 & 1222 & 1251 & 1277 & 1290 \\
\hline 1578 & 1357 & 1369 & 1408 & 1478 & 1549 & 1455 & 1578 & 1599 & 1603 & 1605 & 1696 \\
\hline 1735 & 1799 & 1815 & 1852 & 1899 & 1925 & 1965 & & & & & \\
\hline
\end{tabular}

Table 5. The Maximum Likelihood Estimation of the MDRMW distribution for the Leukemia Data and Standard Errors.

\begin{tabular}{lllcc}
\hline MODEL & $\boldsymbol{q}$ & $\boldsymbol{\beta}$ & $\boldsymbol{b}$ & $\boldsymbol{c}$ \\
\hline MDRM & $0.8091\left(2.37 \times 10^{-2}\right)$ & $1.494 .8\left(1.615 \times 10^{3}\right)$ & $-2214.9\left(6.78 \times 10^{3}\right)$ & $0.9274\left(2.676 \times 10^{-2}\right)$ \\
DRMW & 0.9706 & - & 0.8921 & $(-0197$ \\
\hline
\end{tabular}

Table 6. The AIC and BIC for the fitted distributions

\begin{tabular}{llll}
\hline MODEL & -2Log-Likelihood & AIC & BIC \\
\hline MDRMW & 660.2 & 668.2 & 675.3 \\
DRMW & 745.9 & 751.9 & 757.2 \\
\hline
\end{tabular}

\section{Conclusion}

A new distribution called Marshall-Olkin Discrete Reduced Modified Weibull distribution (MDRMW) was proposed and its properties studied. In this work, the new discrete RMW was used to analyze all the datasets used by Amalki, (2014) in order to properly compare the new distribution with DRMW of Amalki. In addition, two most recent distributions which are Exponentiated Discrete Weibull distribution and Two Parameter discrete Lindley distribution (TDL) proposed by Tassaddaq et al., (2016) were also used to analyze the datasets.

The MDRMW distribution is flexible to model discrete data such as survival data and over-dispersed data. Its hazard function is monotonically decreasing, followed by an increasing shape and upside down bathtub. The closed form expressions for the moments, distribution of order statistics were obtained. Maximum likelihood estimation technique was used to estimate the model parameters.

\section{References}

[1] Almalki, S. J. (2014). Statistical analysis of lifetime data using new modified Weibull distributions, a thesis submitted to the University of Manchester for the degree of Doctor of Philosophy in the Faculty of Engineering and Physical Sciences.

[2] Bebbington, M., Lai, C. D. and Zitikis, R. (2007). A flexible Weibull extension. Reliability Engineering and System Safety, 92, 719-726.

[3] Comtet, L. (1974). Advanced Combinatorics: The art of finite and infinite expansions. Springer.

[4] Marshall, A. W., Olkin, I. (1997). A new method for adding a parameter to a family of distributions with application to the exponential and Weibull families, Biometrika 84(3), 641-652. 
[5] Mudholkar, G. S. and Srivastava, D. K. (1993). Exponentiated Weibull family for analyzing bathtub failure-rate data. Reliability, IEEE Transactions on, 42(2), 299-302.

[6] Nooghabi, M. S., Roknabadi, A. H. R. and Borzadaran, G. M. (2011). Discrete modified Weibull distribution. Metron, LXIX, 207-222.

[7] p-value calculator online:

https://graphpad.com/quickcalcs/PValue1.cfm (2017)
[8] Stein, W. E. and Dattero, R. (1984). A new discrete Weibull distribution. Reliability, IEEE Transactions on, 33(2), 196-197.

[9] Tassaddaq, H., Muhammad, A and Munir A. (2016). A Two Parameter Discrete Lindley Distribution January 2016, Volume 39, Issue 1, pp. 45 to 61 DOI.

[10] Zhang, T. and Xie, M. (2011). On the upper truncated Weibull distribution and its reliability implications. Reliability Engineering and System Safety, 96(1), 194-200. 